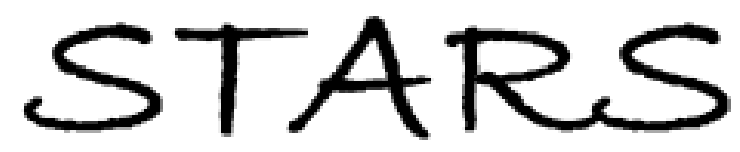

University of Central Florida

STARS

Faculty Bibliography 2000s

Faculty Bibliography

$1-1-2004$

\title{
Electron injection-induced effects in Mn-doped GaN
}

\author{
William Burdett \\ University of Central Florida \\ Olena Lopatiuk \\ University of Central Florida \\ Leonid Chernyak \\ University of Central Florida \\ Martin Hermann \\ Martin Stutzmann
}

See next page for additional authors

Find similar works at: https://stars.library.ucf.edu/facultybib2000

University of Central Florida Libraries http://library.ucf.edu

This Article is brought to you for free and open access by the Faculty Bibliography at STARS. It has been accepted for inclusion in Faculty Bibliography 2000 s by an authorized administrator of STARS. For more information, please contact STARS@ucf.edu.

\section{Recommended Citation}

Burdett, William; Lopatiuk, Olena; Chernyak, Leonid; Hermann, Martin; Stutzmann, Martin; and Eickhoff, Martin, "Electron injection-induced effects in Mn-doped GaN" (2004). Faculty Bibliography 2000s. 4238. https://stars.library.ucf.edu/facultybib2000/4238

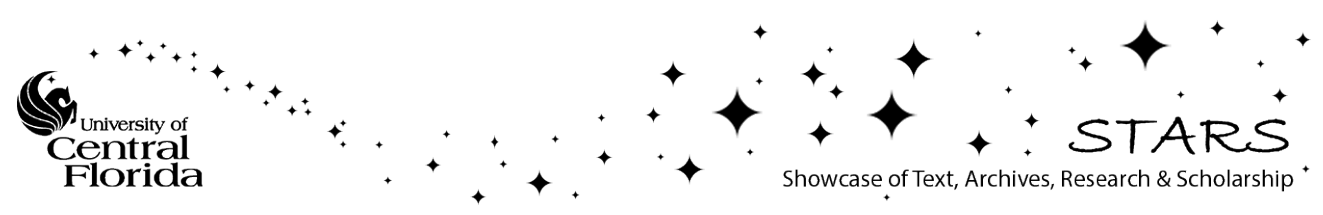




\section{Authors}

William Burdett, Olena Lopatiuk, Leonid Chernyak, Martin Hermann, Martin Stutzmann, and Martin Eickhoff 


\section{Electron injection-induced effects in Mn- doped $\mathrm{GaN}$}

Cite as: J. Appl. Phys. 96, 3556 (2004); https://doi.org/10.1063/1.1780606

Submitted: 22 April 2004 . Accepted: 16 June 2004 . Published Online: 02 September 2004

William Burdett, Olena Lopatiuk, Leonid Chernyak, Martin Hermann, Martin Stutzmann, and Martin Eickhoff

\section{ARTICLES YOU MAY BE INTERESTED IN}

Cathodoluminescence studies of the electron injection-induced effects in GaN Applied Physics Letters 82, 3680 (2003); https://doi.org/10.1063/1.1578514

Study of temperature dependence for the electron injection-induced effects in GaN Applied Physics Letters 81, 1633 (2002); https://doi.org/10.1063/1.1503407

Activation energies of Si donors in GaN

Applied Physics Letters 68, 3144 (1996); https://doi.org/10.1063/1.115805

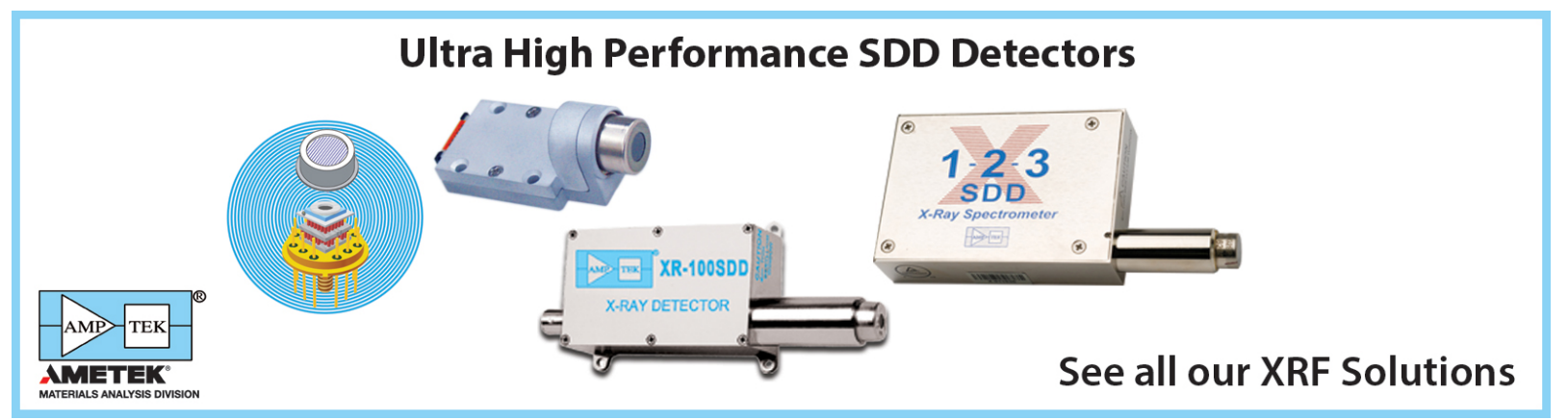

J. Appl. Phys. 96, 3556 (2004); https://doi.org/10.1063/1.1780606 


\title{
Electron injection-induced effects in Mn-doped GaN
}

\author{
William Burdett, Olena Lopatiuk, and Leonid Chernyak ${ }^{\text {a) }}$ \\ Physics Department, University of Central Florida, Orlando, Florida 32816-2385 \\ Martin Hermann, Martin Stutzmann, and Martin Eickhoff \\ Walter Schottky Institute, Technical University Munich, Am Coulombwall 3, D-85748 Garching, Germany
}

(Received 22 April 2004; accepted 16 June 2004)

\begin{abstract}
Electron injection into $\mathrm{Mn}$-doped $\mathrm{GaN}$ resulted in pronounced changes in the minority carrier diffusion length and cathodoluminescence. In particular, multiple-fold decrease of the band-to-band cathodoluminescence intensity was observed in the temperature between -50 and $80^{\circ} \mathrm{C}$. This decrease was accompanied by an increase of the minority carrier diffusion length in the material, measured by electron-beam-induced current. Temperature-dependent cathodoluminescence measurements revealed a recovery of the cathodoluminescence intensity with an activation energy of $360 \mathrm{meV}$. (C) 2004 American Institute of Physics. [DOI: 10.1063/1.1780606]
\end{abstract}

Electron injection into $\mathrm{GaN}$ is important for $p$-type material. Amano et al. ${ }^{1}$ found that highly resistive Mg-doped GaN can be made $p$ type by low energy electron beam irradiation, due of dissociation of hydrogen-Mg complexes. Nakamura $e t a l^{2}$ showed that thermal annealing in vacuum or in nitrogen also activates $\mathrm{Mg}$ acceptors. ${ }^{2}$ Miyachi $e t a{ }^{3}$ and Pearton et $a l^{4}{ }^{4}$ suggest that minority electron injection in $p$-GaN facilitates $\mathrm{Mg}$ thermal activation.

Electron injection into $p$-GaN-either from the electron beam of a scanning electron microscope (SEM) or by the application of an external voltage in a solid state device, ${ }^{5,6}$ increases the minority carrier lifetime and diffusion length. Consistent changes were observed in the material's optical signature. ${ }^{5}$ An electronic model was proposed in which such changes were attributed to charging of metastable centers associated with $\mathrm{Mg}$ doping, ${ }^{5}$ as no effect was observed in $n$-GaN doped with Silicon.

We report on electron injection effects in Mn-doped GaN epitaxial layers. Our results indicate that these effects can be observed in GaN doped with any impurity creating deep levels in the forbidden gap.

Doped and undoped $\mathrm{GaN}$ samples ( $1 \mu \mathrm{m}$ thick) were grown by molecular beam epitaxy on sapphire. Doping resulted in Mn concentrations up to $10^{20} \mathrm{~cm}^{-3}$ and almost intrinsic layers with resistivity $>10^{6} \Omega \mathrm{cm}^{7}$

The experiments were carried out in situ in a Philips XL30 SEM integrated with a Gatan MonoCL3 cathodoluminescence system allowing wavelength-and temperaturedependent optical measurements. ${ }^{6}$ Cathodoluminescence (CL) results were compared with those obtained from the electron-beam-induced current (EBIC) measurements carried out on the same samples. Detailed description of EBIC experiments can be found elsewhere. ${ }^{5}$

EBIC measurements of $\mathrm{GaN}: \mathrm{Mn}$ were performed at room temperature. Figure 1 shows a decay of the EBIC signal (curve 1) as the SEM beam is moved away from the edge of a $30 \mathrm{~nm}$ thick Pt contact evaporated on the sample's top surface. After a single EBIC line-scan was completed (12 s),

${ }^{a)}$ Electronic mail: chernyak@physics.ucf.edu the excitation of the sample with the electron beam was continued for the total time of $\sim 200 \mathrm{~s}$. EBIC measurements were periodically repeated (curves 2,3 ) to extract the minority carrier diffusion length ${ }^{5} L$ as a function of the duration of electron beam irradiation $t$. As displayed in the inset of Fig. 1 , a linear increase of $L$ as a function of $t$ was observed in Mn-doped samples. We have reported a similar behavior in $\mathrm{GaN}$ : Mg. ${ }^{5}$ EBIC measurements carried out on undoped $\mathrm{GaN}$ and $\mathrm{GaN}: \mathrm{Mn}$ layers codoped with Si did not reveal any noticeable increase of $L$ with $t$ suggesting that both the presence of $\mathrm{Mn}$ and the position of the Fermi level (changes with Si codoping) are important for the observed behavior.

Figure 2 shows the room temperature CL spectra for the Mn-doped GaN layer. The full-range CL spectrum is shown in the right inset. Besides a relatively narrow band-to-band peak, it features a broad luminescence band, which remains unaffected by electron irradiation and is attributed to defectinduced recombination across the band gap. In contrast, the intensity of the band-to-band CL, located at $\approx 367 \mathrm{~nm}$, exhibits a continuous decay with $t$ (left inset of Fig. 2). Reduc-

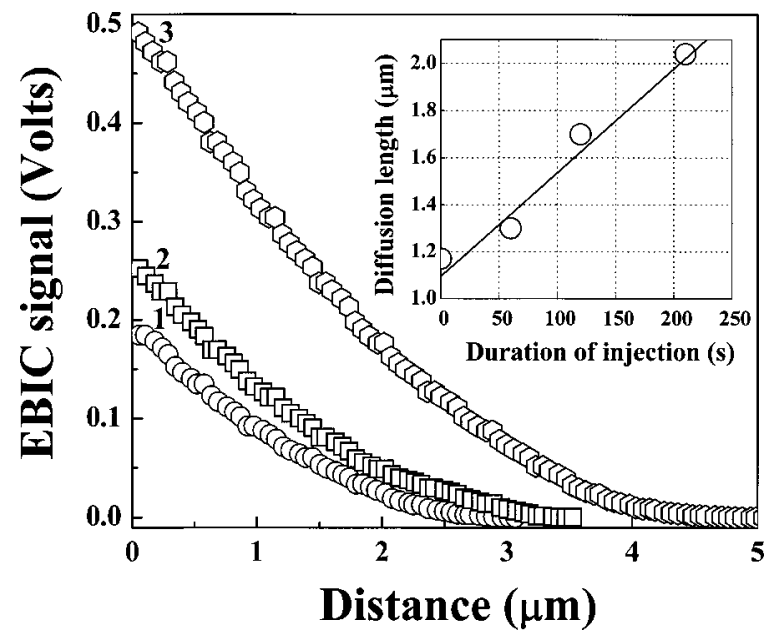

FIG. 1. Room temperature EBIC signal decay for a GaN:Mn sample as a function of distance from the Schottky barrier. Curve 1 corresponds to the initial line-scan, curves 2 and 3 correspond to $t$ of 60 and $210 \mathrm{~s}$, respectively. Inset: $L$ vs $t$ experimental dependence and the linear fit. 


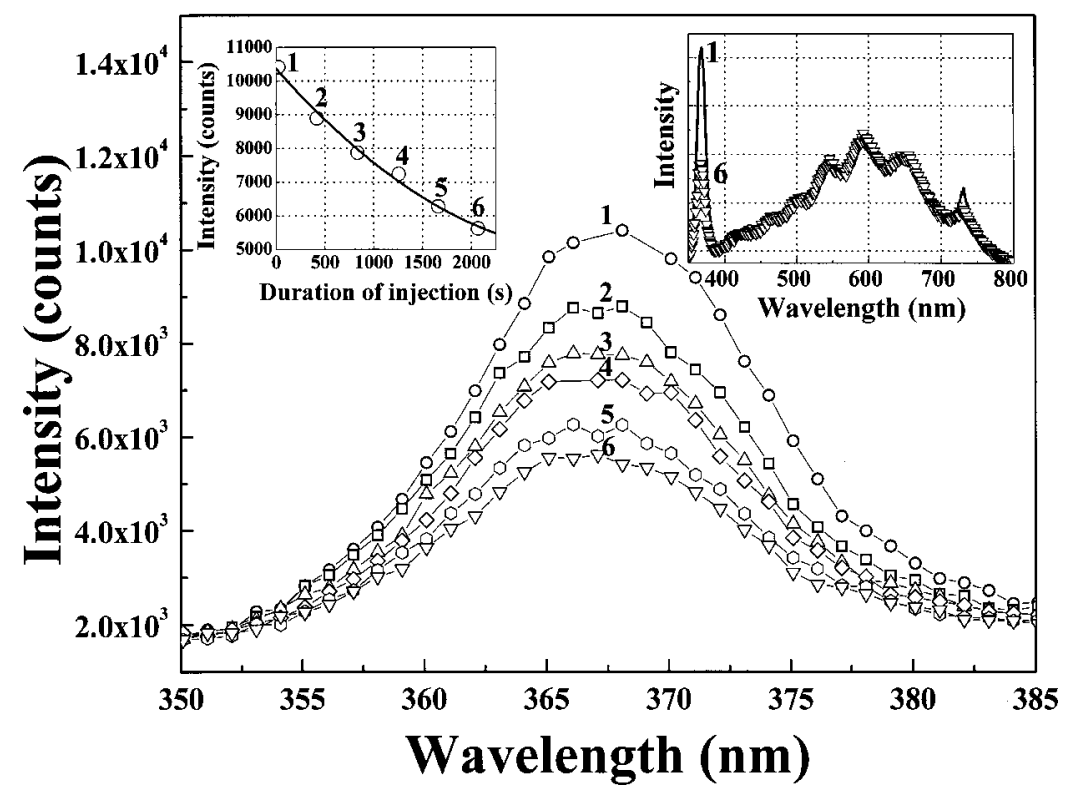

FIG. 2. Room temperature cathodoluminescence measurements after different time intervals of electron injection. The maximum in spectrum 1 nearly corresponds to the preinjection situation (less than $30 \mathrm{~s} \mathrm{time}$ difference). Left inset: Peak CL intensity vs $t$. Right inset: Full range preinjection CL spectrum (1) and $\sim 2100$ s injection spectrum (6).

tion of the Mn concentration to $5 \times 10^{19} \mathrm{~cm}^{-3}$ (from $10^{20} \mathrm{~cm}^{-3}$ ) did not have any effect on the displayed CL behavior.

The minority carrier diffusion length and the band-toband CL intensity are related via the nonequilibrium minority carrier lifetime $\tau$. $L$ increases linearly with $t$, as seen in Fig. 1, and its dependence on the minority carrier lifetime is given by the following expression:

$$
L=\sqrt{D \tau}
$$

where the carrier diffusivity $D$ is unaffected by electron injection. ${ }^{5}$ According to Eq. (1), the inverse CL intensity, which is proportional to the lifetime of carriers in the band ${ }^{7}$, (larger is the value of $\tau$, longer is the nonequilibrium carrier stay in the band, and, as a result, lower is the rate of radiative recombination), should depend quadratically on $t$. This is, indeed, observed in Fig. 3, where the square root of the inverse normalized (with respect to its initial maximum value) intensity, $I^{-1 / 2}$, is plotted versus $t$.

It has been recently shown that Mn forms a deep neutral acceptor level $\left(\mathrm{Mn}^{3+}\right)$ in GaN, with only a very small number of acceptors being ionized $\left(\mathrm{Mn}^{2+}\right){ }^{8} \mathrm{Si}$ codoping results in a shift of the Fermi level position towards the conduction band and to almost complete ionization of $\mathrm{Mn}$ acceptors. Since no dependence of the band-to-band CL or $L$ on electron irradiation was found in $\mathrm{GaN}: \mathrm{Mn}: \mathrm{Si}$ samples and undoped GaN layers, it can be concluded that the effects observed in $\mathrm{GaN}$ : Mn are caused by the presence of neutral $\mathrm{Mn}$ acceptors in the initially nonirradiated samples.

Temperature dependent $\mathrm{CL}$ measurements in the range between $-50^{\circ} \mathrm{C}$ and $80^{\circ} \mathrm{C}$ were carried out to further illuminate the possible physical origin of the experimental findings. Figure 3 shows the dependence of $I^{-1 / 2}$ on $t$ at different temperatures. The slopes of the linear dependencies in Fig. 3 are the rates $R$ for CL decrease. Evidently, an increase in temperature results in a decrease of $R$, suggesting existence of thermally activated process, which counteracts the effect of electron irradiation, thereby leading to a decrease of $\tau$. The activation energy of $360 \mathrm{meV}$ for this process was cal- culated as in Ref. 5 from the Arrhenius plot shown in the inset of Fig. 3. The factor of 2 before the thermal energy $k T$ comes from the two competing factors: $R$ dependence on duration of electron injection $\left[\sim \exp \left(\Delta E_{A} / \mathrm{kT}\right)\right]$ and on temperature $\left[\sim \exp \left(-\Delta E_{A} / 2 \mathrm{kT}\right)\right]^{9}{ }^{9} \Delta E_{A}$ for both competing factor are close to each other.

The model summarized in Fig. 4 follows the defect molecule picture for $\mathrm{Mn}^{3+}$ in $\mathrm{GaN}^{10}{ }^{10}$ Initially, Mn impurities are present in the neutral acceptor state $\mathrm{Mn}^{3+}$, characterized by two filled $e_{\uparrow}$ orbitals and three $t_{2 \uparrow}$ orbitals [left side of Fig. 4(a).] While electrons occupy two of the latter orbitals, the third one is empty. Recombination of nonequilibrium electron-hole pairs created by SEM electron beam causes the band-to-band cathodoluminescence. In addition, electron beam injection can lead either to electron transfer from $e_{\uparrow}$ to an empty $t_{2 \uparrow}$ orbital or, competitively, to direct ionization of the $\mathrm{Mn}^{3+}$ state to $\mathrm{Mn}^{2+}$ [process (1) in Fig. 4(a)].

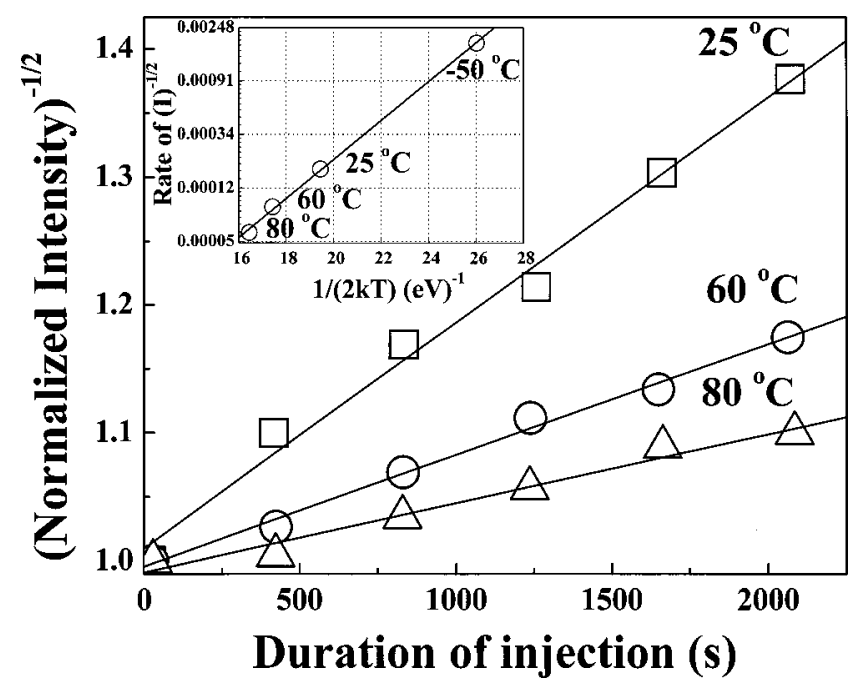

FIG. 3. Variable temperature dependence for the square root of inverse normalized intensity on the time of electron injection. The rate at every temperature is obtained from the slope of a linear fit. Inset: Temperature dependence for the rate of the square root of inverse normalized intensity (open circles) and the fit. 
(a)

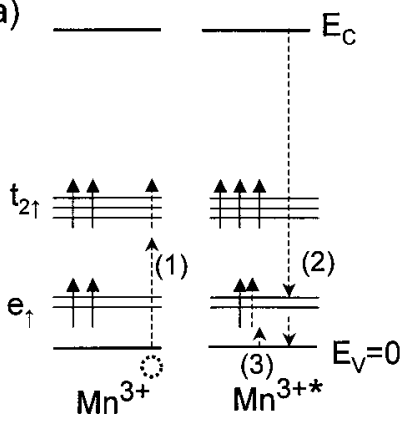

(b)

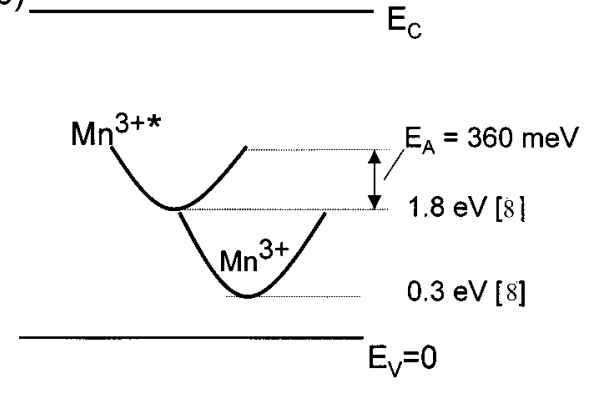

FIG. 4. Possible mechanism for the observed electron injection-induced effects. (1) Ionization of $\mathrm{Mn}^{3+}$ to $\mathrm{Mn}^{2+}$ due to a SEM beam excitation and a consequent electron [dashed arrow in (a), left] transition to a vacant $t_{2 \uparrow}$ orbital. (2) Band-to-impurity recombination through a vacant $\mathrm{Mn}^{3+*} e_{\uparrow}$ orbital [(a), right]. (3) Thermally activated hole emission (electron capture) from an $e_{\uparrow}$ orbital of the $\mathrm{Mn}^{3+*}$ state to the valence band. This leads to ionization of $\mathrm{Mn}^{3+*}$ to $\mathrm{Mn}^{2+}$ and, thus, to a suppression of (2).
In the first case, electron transfer causes generation of the energetically higher $\mathrm{Mn}^{3+*}$ state (one electron on $e_{\uparrow}$ orbital and three electrons on $t_{2 \uparrow}$ orbitals), shown on the right side of Fig. 4(a). This lead to activation of a nonradiative recombination channel (2) via a vacant $e_{\uparrow}$ orbital and therefore to a decrease in the intensity of the band-to-band cathodoluminescence, as is seen in Fig. 2. Here, we have to assume that the transition (2) in Fig. 4(a) dominates over the bandto-band transition after the electron irradiation is started, while a recombination through the $t_{2 \uparrow}$ orbitals of the $\mathrm{Mn}^{3+}$ ground state [cf Fig. 4(a), left] is not allowed. Although the exact reason for this is unclear at the moment, the experimental evidence exists. Note that the process of $\mathrm{Mn}^{3+}$ ionization to $\mathrm{Mn}^{2+}$ creates a hole in the valence band and, thus, would not lead to the observed increase of the minority carrier diffusion length. This is because the nonequilibrium electrons of the conduction band, induced by electron beam irradiation, would then recombine with the nonequilibrium holes, available in the valence band, leading to a decrease of the nonequilibrium carrier lifetime and $L$ in agreement with the Eq. (1).

Because the Fermi level in $\mathrm{GaN}: \mathrm{Mn}$ samples is close to the middle of the band gap, the carrier diffusion length determined from EBIC most likely represents the effective diffusion length for the nonequilibrium electron-hole pairs. Therefore, the longer the nonequilibrium carrier lifetime, the longer $L$ (cf. Fig. 1), and smaller is the number of recombination events including those through the band-to-band transition. As a result, the band-to-band CL continues to decrease with increasing duration of electron injection.

Two different mechanisms can cause a reactivation of the band-to band channel when the temperature is increased. A thermally activated charge neutral transition of a metastable $\mathrm{Mn}^{3+*}$ to a $\mathrm{Mn}^{3+}$ state [Fig. 4(b)] establishes the initial situation and quenches the electron irradiation effects. Up to now, such a transition has not been reported in the literature.
Note that a thermally activated hole emission from an $e_{\uparrow}$ orbital of the $\mathrm{Mn}^{3+*}$ state to the valence band is also likely to occur [process (3) in Fig. 4(a) right]. According to Ref. 8, this process exhibits an activation energy of $300 \mathrm{meV}$. At the same time, the temperature-dependent rate of CL decrease observed in this work shows the activation energy $E_{A}$ $=360 \mathrm{meV}$. The relatively large difference between the above activation energies indicate that a charge neutral thermally activated transition from $\mathrm{Mn}^{3+*}$ to $\mathrm{Mn}^{3+}$ might occur.

The obtained results indicate the role of Mn-related states in the forbidden gap. Additional studies are under way to understand the kinetics of $L$ and CL relaxation to the preinjection levels after an electron beam irradiation is stopped. So far lasting electron injection-induced changes, similar to $\mathrm{Mg}$-doped $p-\mathrm{GaN},{ }^{5,6}$ were observed.

\section{ACKNOWLEDGMENTS}

Support of NSF, PRF, and Deutsche Forschungsgemeinschaft is acknowledged.

${ }^{1}$ H. Amano, N. Sawaki, I. Akasaki, and Y. Toyoda, Appl. Phys. Lett. 48, 353 (1986).

${ }^{2}$ S. Nakamura, T. Mukai, M. Senoh, and N. Iwasa, Jpn. J. Appl. Phys. 31, L139 (1992).

${ }^{3}$ M. Miyachi, T. Tanaka, Y. Kimura, and H. Ota, Appl. Phys. Lett. 72, 1101 (1998).

${ }^{4}$ S. J. Pearton, J. C. Zolper, R. J. Shul, and F. Ren, J. Appl. Phys. 86, 1 (1999).

${ }^{5}$ L. Chernyak and W. Burdett, MRS Spring Meeting Proceedings, 2003.

${ }^{6}$ L. Chernyak, W. Burdett, M. Klimov, and A. Osinsky, Appl. Phys. Lett. 82, 3680 (2003).

${ }^{7}$ S. M. Sze, Semiconductor Devices Physics and Technology (Wiley, New York, 1985).

${ }^{8}$ T. Graf, M. Gjukic, M. S. Brandt, M. Stutzmann, and O. Ambacher, Appl. Phys. Lett. 81, 5159 (2002).

${ }^{9}$ W. C. Burdett, O. Lopatiuk, A. Osinsky, S. J. Pearton, and L. Chernyak, Superlattices Microstruct. 34, 55 (2004).

${ }^{10}$ T. Graf, S. T. B. Goennenwein, and M. S. Brandt, Phys. Status Solidi B 239, 277 (2003) 